\title{
The ATLAS ITk strip detector system for the High Luminosity LHC upgrade
}

\author{
John Stakely Keller, on behalf of the ATLAS Collaboration
}

Carleton University, 1125 Colonel By Drive, Ottawa, Canada

\section{A R T I C L E I N F O}

\section{Keywords:}

HL-HLC

\section{ATLAS}

Tracker

Strip

Semiconductor

Silicon

\begin{abstract}
A B S T R A C T
The ATLAS experiment at the Large Hadron Collider is currently preparing major detector upgrades to meet the requirements of the High Luminosity LHC (HL-LHC), scheduled to begin in 2026. With the radiation damage and high density of tracks expected at the HL-LHC, the current Inner Detector would be inoperable, and will instead be replaced by an all-silicon Inner Tracker (ITk). The ITk consists of two sub-systems: a pixel detector close to the beam pipe, and a strip detector at larger radii. This paper presents results from an extensive design and prototyping effort for the ITk strip detector, including studies of the sensors, electronics, and support structures. The performance of the detector has been validated through testbeams, simulations, and thermal and electrical prototypes. Plans for the forthcoming production phase are also presented.
\end{abstract}

\section{Introduction}

The ATLAS detector at the Large Hadron Collider has been operating successfully for nearly a decade, recording more than $175 \mathrm{fb}^{-1}$ of pp collision data. From 2024 to 2026, the LHC will be upgraded to the High Luminosity LHC (HL-LHC), which is expected to deliver instantaneous luminosity up to $7.5 \times 10^{34} \mathrm{~cm}^{-2} \mathrm{~s}^{-1}$, and an integrated luminosity up to $4 \mathrm{ab}^{-1}$ per experiment. This will provide unprecedented opportunities for precise measurements in the Higgs and electroweak sectors, as well as searches for physics beyond the Standard Model. However, the HL-LHC will also pose significant challenges to the experiments. Most notably, the pileup will reach an average of 200 interactions per bunch crossing, and hadron fluences of up to $2 \times 10^{16} \mathrm{n}_{\mathrm{eq}} / \mathrm{cm}^{2}$ will cause significant radiation damage.

The current Inner Detector at ATLAS, designed for LHC conditions, would not be able to cope with the high track density or radiation damage of the HL-LHC. Instead, a new detector will be installed, known as the Inner Tracker (ITk). The ITk will be an all-silicon tracker, consisting of a pixel detector [1] in the region closest to the beam pipe, and a strip detector at higher radii [2]. The layout of the ITk is shown in Fig. 1. The pixel detector consists of five barrel layers, with inclined sensors in the forward region, as well as an endcap with individually located rings. The strip detector consists of four barrel layers and six disks in each endcap. The ITk will extend the tracking coverage of ATLAS from $|\eta|<2.5$ to $|\eta|<4$.0. With increased granularity, reduced material, and more radiation-hard sensors, the ITk is expected to maintain or improve the tracking performance in spite of the more challenging environment.

\section{Overview of the ITk strip detector}

\subsection{Sensors and modules}

In order to facilitate a multi-site construction effort, the ITk strip detector employs a highly modular design: the smallest structures (modules) are built into larger structures (staves and petals), which are built into the still larger structures (cylinders and disks) that make up the detector. The most basic unit, a module, consists of a single strip sensor, one or two flex hybrid circuit boards, and a powerboard. An example of a prototype endcap module is shown in Fig. 2.

The silicon strip sensors must withstand a hadron fluence of up to $1.6 \times 10^{15} \mathrm{n}_{\mathrm{eq}} / \mathrm{cm}^{2}$ (including a safety factor of 1.5 ). For this reason, $\mathrm{n}^{+}$-in-p Float Zone sensors are used [3], which do not suffer from the radiation-induced type inversion seen in n-bulk sensors. Strip isolation is improved using p-stop implants. Extensive irradiation campaigns have been carried out to ensure the sensor performance at the end of lifetime. Fig. 3 shows the collected charge as a function of the fluence for different forms of irradiation, measured using beta sources. The results indicate that at the maximum expected fluence an observed signal of more than 10,000 electrons can still be seen. This can be compared to noise values which are expected to be well below 1000 electrons.

The sensors are $320 \mu \mathrm{m}$ thick, and made on 6-inch wafers. Sensors in the barrel are approximately $10 \times 10 \mathrm{~cm}^{2}$, while in the endcap, six different annular shapes are used in order to fit the nearly trapezoidal shape of the petals. Sensors contain either two or four rows of strips, with the strip length varying from 18.1 to $60.2 \mathrm{~mm}$, depending on the expected track density. The strip pitch is $75.5 \mu \mathrm{m}$ for barrel sensors, while it varies from approximately 70 to $80 \mu \mathrm{m}$ in the endcap.

\footnotetext{
Copyright 2019 CERN for the benefit of the ATLAS Collaboration. Reproduction of this article or parts of it is allowed as specified in the CC-BY-4.0 license. E-mail address: jkeller@physics.carleton.ca.
} 


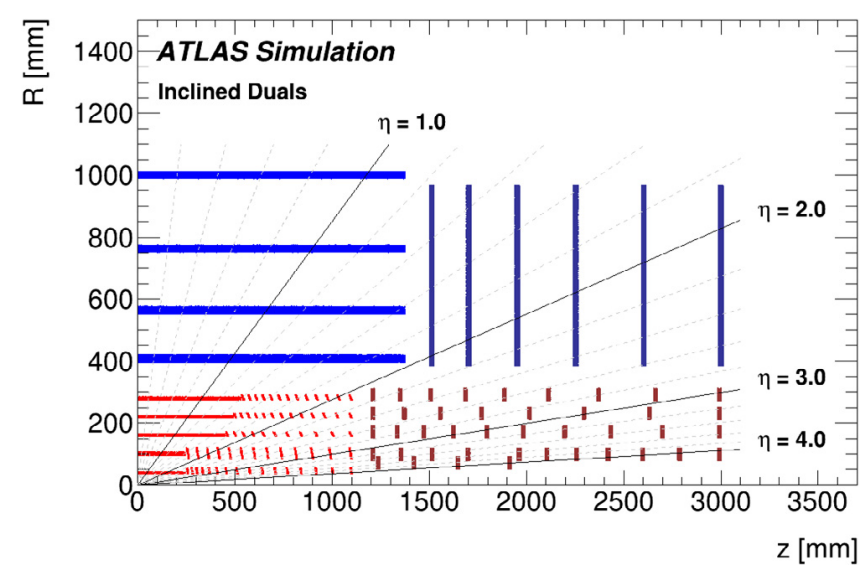

Fig. 1. Layout for the ATLAS ITk upgrade [1]. The pixel subsystem is shown in red while the strip subsystem is shown in blue. One quarter of the detector is shown. (For interpretation of the references to color in this figure legend, the reader is referred to the web version of this article.)

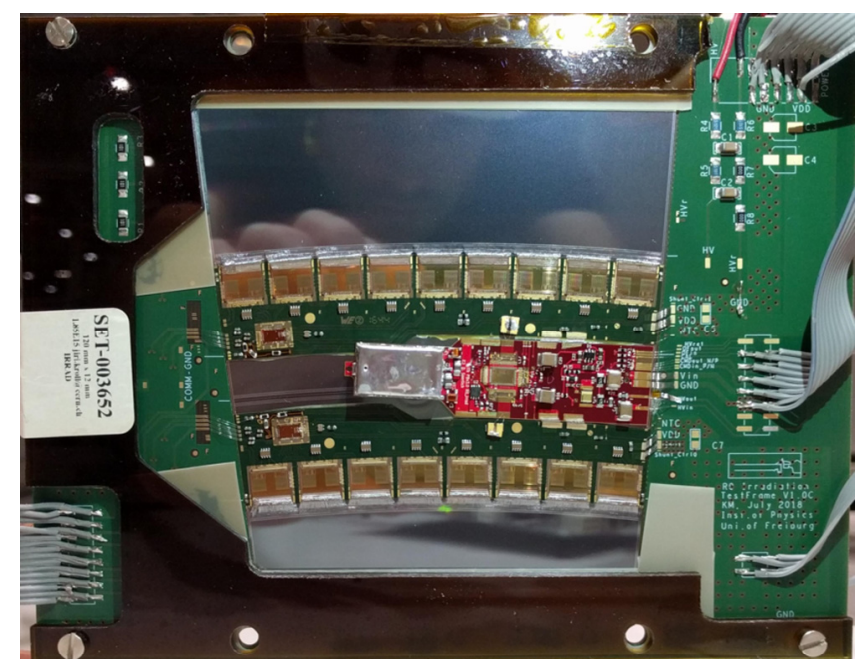

Fig. 2. An example of an ITk strip endcap module on a testframe. Two green hybrids are glued to the sensor, each containing eight or nine ABC130 ASICs and one HCC. A red powerboard is also glued to the sensor, including a DC-DC converter in a rectangular metallic shield. (For interpretation of the references to color in this figure legend, the reader is referred to the web version of this article.)

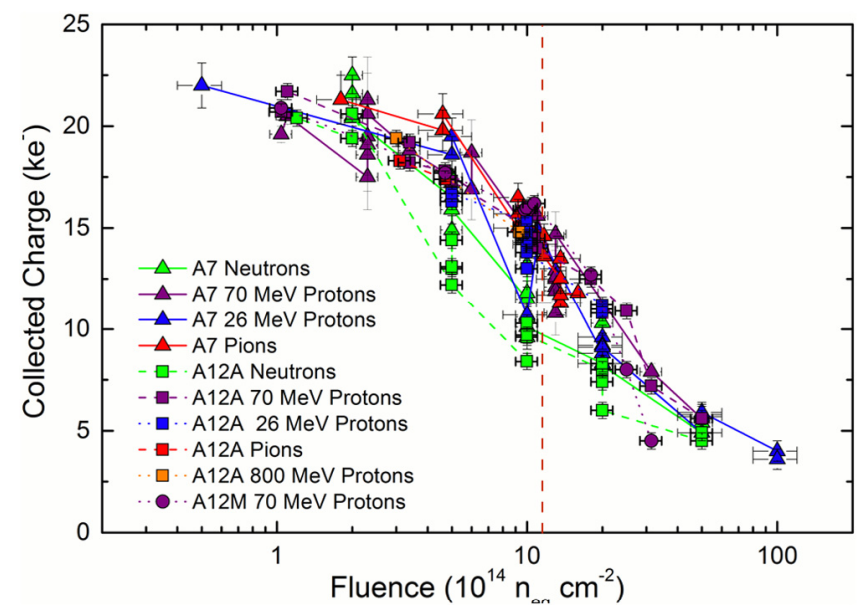

Fig. 3. Collected charge in beta source measurements for various prototype miniature sensors, after irradiation of different types and to different fluences [2]. The sensors are biased to $500 \mathrm{~V}$. A7 and A12 refer to sensors delivered in 2007 and 2012, respectively, while $\mathrm{A} 12 \mathrm{M}$ includes stereo rotated strips. The dashed line indicates the approximate maximum fluence expected at the end of lifetime.
As both sides of support structures are populated with modules, 3-dimensional spacepoint reconstruction is achieved by introducing a stereo rotation between the strips of back-to-back sensors. In the barrel, the sensors are rotated by $26 \mathrm{mrad}$, for a total stereo angle of $52 \mathrm{mrad}$. In the endcap, space constraints prevent rotation of the sensors, so the strips themselves are skewed to point slightly off-center, resulting in a stereo angle of $40 \mathrm{mrad}$.

The hybrid(s) and powerboard are glued directly on top of the sensor to create a module. Hybrids contain a number of front-end ASICs, the ATLAS Binary Chips or ABCstars, as well as one Hybrid Control Chip, or HCCstar [4]. These are described further in the next section. The powerboard contains a DC-DC converter, the radiationhard bPOL12V, which reduces the input voltage from $11 \mathrm{~V}$ to $1.5 \mathrm{~V}$ as needed by the ASICs. The use of a DC-DC converter reduces ohmic losses in the cables allowing a reduction of the material budget [5]. In addition, the powerboard contains an Autonomous Monitoring and Control chip (AMAC), as well as the high voltage bias filter and switch [6].

\subsection{ASICs and electronics}

The ABCstar front-end chip is built from $130 \mathrm{~nm}$ CMOS technology, and uses analogue amplification and shaping to provide binary output. Each chip has 256 input channels, split among two rows. Data is sent in parallel from each of the ABCstars on one hybrid to the HCCstar, which serves as the interface between the module and the local support. Final versions of the ABCstar and HCCstar chips became available in late 2018. For this reason, most prototyping studies so far, including those in Section 3.1, have been done with a previous chipset, the ABC130 and HCC. These chips also used $130 \mathrm{~nm}$ technology, but employed a serial readout scheme.

Every HCCstar on a stave or petal sends data to an End-of-Substructure (EoS) card, at a rate of up to $640 \mathrm{Mbps}$. The EoS also sends trigger, timing, and control (TTC) information to the HCCstars at a rate of 120 Mbps. Additionally, detector control signals and bias high voltage are sent from the EoS to the powerboard. The EoS is connected to the modules via a polyimide bus tape. The EoS contains optical transceivers which send data to the off-detector electronics at up to 8.96 Gbit/s [7].

\subsection{Support structures}

ITk strip modules are glued onto staves and petals in the barrel and endcap, respectively. A schematic of a stave or petal is shown in Fig. 4. These are low-mass support structures based on carbon fiber honeycomb [8]. Cooling is provided by bi-phase $\mathrm{CO}_{2}$ circulated through Titanium cooling pipes. The pipes are surrounded by high-conductivity thermal foam. The bus tapes providing electrical connection between the modules and the EoS card are co-cured to the core.

The staves and petals are designed to be inserted into the global support structures, cylinders and disks. This allows testing of the multimodule components prior to assembly into the largest structures. The global supports are made primarily from carbon fiber, to reduce material while providing support across a wide temperature range. All services are connected at the end of the structures, to reduce the material budget inside the tracker.

\section{Prototyping studies}

\subsection{Testbeams}

The performance of prototype modules has been extensively studied using high energy testbeam facilities. Two such campaigns were undertaken in 2018, using $4 \mathrm{GeV}$ electrons from the DESY-II facility, and $120 \mathrm{GeV}$ pions from the CERN SPS facility. At DESY, the first longstrip barrel modules were tested, as was the first double-sided endcap 


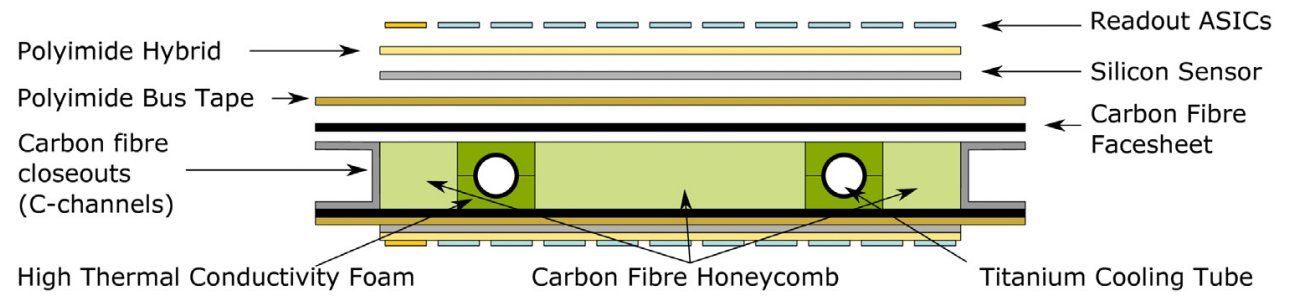

Fig. 4. Schematic of the components of a stave or petal core. The figure is not to scale.

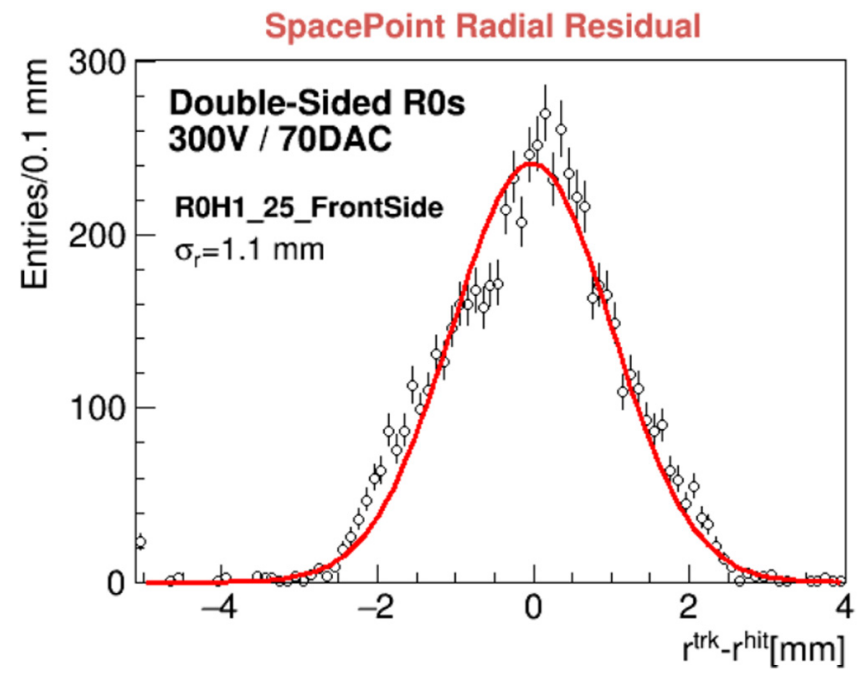

Fig. 5. Residuals for the reconstructed track position in the direction along the strips, using the stereo rotation in a double-sided endcap structure [9].

structure (two-modules glued on either side of a carbon fiber core). At CERN, an endcap module including a powerboard was irradiated to a fluence of $1.63 \times 10^{15} \mathrm{n}_{\mathrm{eq}} / \mathrm{cm}^{2}$ at the PS-IRRAD facility, and subsequently tested at the SPS testbeam facility. In both cases, tracking was provided by an EUDET-style telescope [10], with reconstruction performed using the EUTelescpe framework [11].

Both the long-strip modules and the double-sided endcap structure showed good performance, with a wide range of operating thresholds showing efficiency above $99 \%$ and noise occupancy below $0.1 \%$. This indicates the possibility for excellent tracking at the beginning of lifetime. Additionally, having two modules back-to-back allows for a demonstration of the 3-D spacepoint reconstruction using the stereo angle. Fig. 5 shows the residuals for the reconstructed track position in the direction along the strips. The results are consistent with the expected distance of 1.8 to $2 \mathrm{~mm}$ between strip crossings (calculated as the strip pitch divided by the sine of the stereo angle). Analysis of data from the irradiated endcap module is ongoing; however, demonstration of the operation of the module after receiving more than the maximum expected fluence in a short time period gives confidence for the operability of the detector at the end of lifetime.

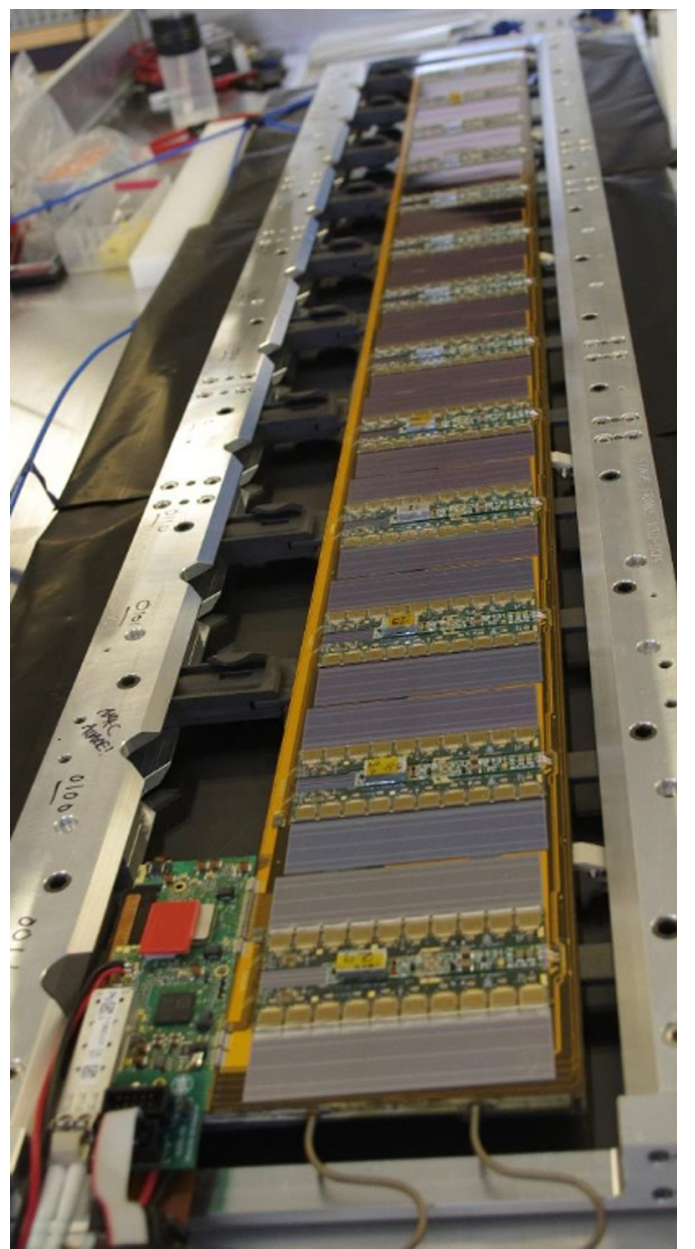

Fig. 7. Semi-electrical stave using two real modules and 11 dummy modules.

\subsection{Thermal studies}

The thermal properties of staves and petals at the end of lifetime have been evaluated using Finite Element Analysis (FEA). These studies indicate that thermal runaway can be avoided if the evaporation

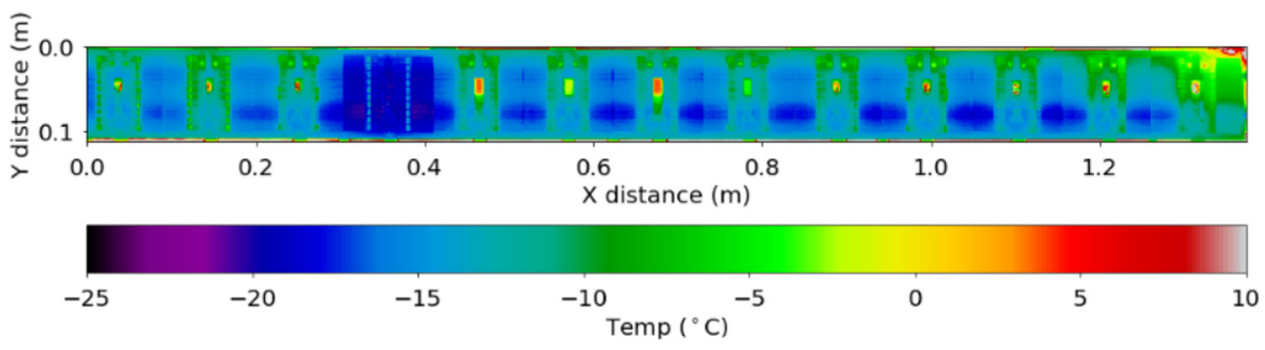

Fig. 6. Measured temperature on a thermo-mechanical stave. The fourth module from the left has been intentionally disabled. 
temperature of the $\mathrm{CO}_{2}$ is less than $-15 \mathrm{C}$. As the baseline temperature is $-35 \mathrm{C}$, the studies show significant headroom.

The FEA simulations have been validated using thermo-mechanical prototype staves and petals. These prototypes have real carbon cores, but plain silicon "sensors" and heating elements to mimic the ASICs. They are painted black to ensure constant emissivity. Fig. 6 shows the measured temperature on a thermo-mechanical stave. The results agree with the FEA prediction within a few tenths of a degree.

\subsection{Electrical staves}

The first fully populated staves have recently been constructed. The first of these, shown in Fig. 7, uses a combination of real modules and "dummy" modules (built with plain silicon instead of real sensors), while the second is made entirely from real modules. An increase in noise of around $10 \%$ is seen in the modules after placing them on the stave. Nonetheless, the noise remains acceptably low. The next steps include moving to the final chip set, multi-stave systems tests, and a semi-electrical petal (using a combination of real and dummy modules).

\section{Preparations for production}

With nearly all design decisions made, and excellent performance demonstrated in many areas, the project is transitioning from the research and development phase into the production phase. In total, 392 staves and 384 petals must be constructed, comprising 17888 modules. The effort will be shared among many institutes across the globe. Preproduction will begin in summer 2019 , where $5 \%$ of the material will be delivered to establish procedures and qualify construction sites. Particular attention is paid to the quality control and quality assurance procedures, ensuring a highly-functioning detector. This phase will be followed by production, which will last until 2024. At this point the detector will be installed and commissioned in preparation for collision data in 2026.

\section{Conclusion}

The ATLAS ITk strip detector will be required to provide high tracking performance in the harsh environment of the HL-LHC, even after significant radiation damage. A major research and development program has resulted in a detector design that is capable of meeting these challenging requirements. This has been demonstrated in recent prototype studies, including testbeams, thermal simulations and prototypes, and fully populated staves. These efforts will continue in the near future, in particular incorporating the final set of ASICs. In parallel much of the focus is shifting to preparations for production, which will last from 2019 until 2024 and involve a large, multi-national effort.

\section{Acknowledgments}

Some of the measurements leading to these results have been performed at the Test Beam Facility at DESY Hamburg (Germany), a member of the Helmholtz Association (HGF); and at the CERN SPS Test Beam Facility. We gratefully thank the operators of these facilities. This project has received funding from the European Union's Horizon 2020 research and innovation programme under grant agreement No. 654168.

\section{References}

[1] ATLAS Collaboration, Technical Design Report for the ATLAS Inner Tracker Pixel Detector, CERN-LHCC-2017-021, ATLAS-TDR-030, 2017.

[2] ATLAS Collaboration, Technical Design Report for the ATLAS Inner Tracker Strip Detector, CERN-LHCC-2017-005, ATLAS-TDR-025, 2016.

[3] Y. Unno, et al., Development of $n+-$-in-p large-area silicon microstrip sensors for very high radiation environments - ATLAS12 design and initial results, Nucl. Instrum. Methods Phys. Res. A 765 (2014) 80-90.

[4] K. Mahboubi, et al., The front-end hybrid for the ATLAS HL-LHC silicon strip tracker, J. Instrum. 9 (2014) C02027.

[5] A. Affolder, et al., DC-DC converters with reduced mass for trackers at the HL-LHC, J. Instrum. 6 (11) (2011) C11035.

[6] E.G. Villani, et al., HVMUX, the high voltage multiplexing for the ATLAS tracker upgrade, J. Instrum. 10 (01) (2015) C01041.

[7] P. Moreira, et al., The GBT Project, 2009, https://cds.cern.ch/record/1235836.

[8] S. Diez, Silicon strip staves and petals for the ATLAS Upgrade tracker of the HL-LHC, Nucl. Instrum. Methods Phys. Res. A 699 (2013) 93-96.

[9] F. Ruehr, Test beam results of prototype modules for the ATLAS ITk Strip detector, in: 7th Beam Telescopes and Test Beams Workshop, 18 Jan 2019.

[10] H. Jansen, et al., Performance of the EUDET-type beam telescopes, EPJ Tech. Instrum. 3 (1) (2016) 7.

[11] EUTelescope, A generic pixel telescope data analysis framework, http:// eutelescope.web.cern.ch/. (Accessesed March 01, 2019). 